\title{
Numerical analysis of heterogeneous mathematical model of elastic body with thin inclusion by combined BEM and FEM
}

\author{
Dyyak I. I. ${ }^{1}$, Rubino B. ${ }^{2}$, Savula Ya. H. ${ }^{1}$, Styahar A. O. ${ }^{1,2}$ \\ ${ }^{1}$ Ivan Franko Lviv National University of Lviv, \\ 1 Universytetska Str., 79000, Lviv, Ukraine \\ ${ }^{2}$ University of L'Aquila, \\ via Vetoio, loc. Coppito, 67100, L'Aquila, Italy
}

(Received 30 September 2019; Accepted 16 October 2019)

\begin{abstract}
This article dwells upon the multiscale elastic structures consisting of matrix medium and thin coatings or inclusions. The matrix medium is described by the equations of classical elasticity theory, while Timoshenko shell theory is used for the description of the thin parts of the structure. On the interface between media, perfect contact conditions are assumed to hold. The coupled algorithm is developed, based on the boundary element method in the matrix part and on the high order finite element method in the thin parts of the structure. The two methods are coupled using a domain decomposition approach. Two numerical examples are considered to illustrate the proposed approach: a Girkmann-type problem and an elastic structure with a thin inclusion. The dependence of the displacement and the stress-strain state on the different shell shapes in the first example and on the inclusion thicknesses in the second example are analyzed.
\end{abstract}

Keywords: finite element method, boundary element method, Timoshenko shell theory, elasticity theory.

2000 MSC: 74-xx; 65N30; 65N38; 65N55

UDC: $531 ; 519.6$

DOI: $10.23939 / \mathrm{mmc} 2019.02 .239$

\section{Introduction}

Contact problems of elasticity theory for structures that contain thin inclusions or coatings arise naturally in applications in engineering and various fields of science. These problems are difficult to analyze both numerically and analytically. In order to simplify the analysis of these types of problems, heterogeneous mathematical models $[1,2]$ or multiscale models $[3,4]$ are typically used. Multiscale modeling is often used while combining mixed-dimensional models [5] (D-adaptive modeling [6]). In addition, multiscale approaches can be applied for coupling processes of different physical scales [4]. The strength and reliability of the elements of various engineering structures are ensured by the detailed information about the stress-strain state, which is practically impossible to obtain without the use of multiscale modeling.

In the case of heterogeneous modeling for contact problems for structures that contain thin inclusions, different hierarchical models are used for the description of the stress-strain state of the inclusions [7]. These models are obtained by assuming particular behavior of displacement and stress fields inside the inclusions, taking into account the thickness of the inclusions. Such models include equations of various theories of plates and shells [7-9]. In this article we consider plain deformation of the structure containing a thin inclusion. In this case, different types of equations are used in different parts of structure for the description of physical phenomena; in each subdomain, particular numerical approaches are applied for the analysis of the undergoing processes. For the description of the stressstrain state of the elastic body the equations of the linear elasticity theory in the form of boundary integral equations (BIE) are used [10]. The stress-strain state of the thin inclusion is modeled using 
the equations of linear Timoshenko shell theory, which for the problem under consideration is a system of ordinary differential equations (ODE) from the mathematical point of view [6].

Systems of BIE and ODE are coupled using special contact conditions on the interface [11,12], which one may consider as a variant of multipoint constraint method [3]. Numerical treatment of the problem is based on the domain decomposition method (DDM), namely Dirichlet-Neumann scheme [13]. This allows us to obtain a solution using direct boundary element method [6,10]. In order to get the numerical solution of ODE in its weak form we apply the finite element method (FEM).

The aim of this paper is to show that heterogeneous modeling, boundary element method (BEM), FEM and DDM can be used to build an efficient approach for the structures that contain thin inclusions and coatings. In the first section, we formulate a heterogeneous mathematical model as a coupled system of integral and differential equations. In the second section, theoretical results on the questions of the well-posedness of the mathematical problem corresponding to the heterogeneous model are addressed. The third section deals with algorithm of the numerical solution of the problem, including BEM, FEM and DDM. In the fourth section the results of numerical experiments are analyzed. One of the examples under analysis is the Girkmann type problem which is analyzed in detail in [14].

\section{Mathematical model of an elastic body with a thin inclusion}

Let us consider an elastic body bounded by the domain $\Omega=\Omega_{1} \cup \Omega_{2}$ with Lipschitz boundaries $\Gamma_{1}$ and $\Gamma_{2}$ respectively (see Fig. 1).

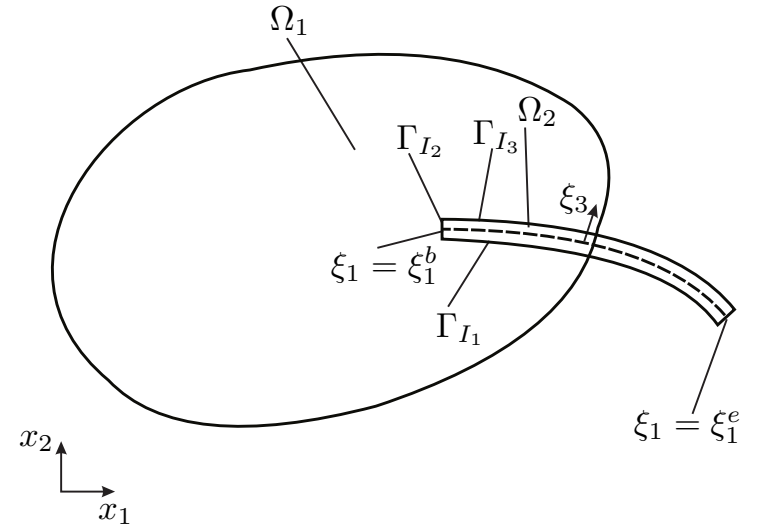

Fig. 1. Physical structure under consideration.

Let us define the displacement vector $u=\left(u_{1}, u_{2}\right)$ in $\Omega_{1}$ and $v=\left(v_{1}, w, \gamma_{1}\right)$ in $\Omega_{2}$. Furthermore, we assume the matrix and inclusion to be isotropic with $E_{1}$ and $E_{2}$ being Young's moduli, $\nu_{1}, \nu_{2}$ being Poisson's ratios. The equilibrium equations in $\Omega_{1}$ are given by [10]

$\frac{1}{2} u_{j}\left(x_{0}\right)=\int_{\Gamma_{1}}\left[t_{i}(x) G_{i j}\left(x, x_{0}\right)-F_{i j}\left(x, x_{0}\right) u_{i}(x)\right] d \Gamma(x)$,

which hold for $x_{0} \in \Gamma_{1}, i, j=1,2$, where $u(x)=$ $\left(u_{1}(x), u_{2}(x)\right)$ and $t(x)=\left(t_{1}(x), t_{2}(x)\right)$ are the vectors of displacements and tractions in $\Omega_{1}$ along $x_{1}$ axis and $x_{2}$ axis; $G_{i j}(x, \xi)=C_{1}\left(C_{2} \delta_{i j} \ln r-\frac{y_{i} y_{j}}{r^{2}}\right)$ is the Green's function; $F_{i j}(x, \xi)=\frac{C_{3}}{r^{2}}\left(C_{4}\left(n_{j} y_{i}-n_{i} y_{j}\right)+\left(C_{4} \delta_{i j}+\right.\right.$ $\left.\frac{2 y_{i} y_{j}}{r^{2}}\right) y_{k} n_{k}$ is the co-normal derivative of Green's function, $r^{2}=y_{i} y_{i}, y_{i}=x_{i}-\xi_{i}, C_{1}=-\frac{1}{8 \pi \mu_{1}\left(1-\nu_{1}\right)}$, $C_{2}=3-4 \nu_{1}, C_{3}=-\frac{1}{4 \pi\left(1-\nu_{1}\right)}, C_{4}=1-2 \nu_{1}, n_{1}, n_{2}$ are the components of the outer normal vector for $\Omega_{1}, \mu_{1}=\frac{E_{1}}{2\left(1+\nu_{1}\right)}$ is the shear modulus, $E_{1}$ is the Young's modulus, $\nu_{1}$ is the Poisson's ratio.

In order to describe the stress-strain state inside $\Omega_{2}$ we use the equations of Timoshenko shell theory of the form [15]:

$$
\begin{aligned}
& -\frac{1}{A_{1}} \frac{d T_{11}}{d \xi_{1}}-k_{1} T_{13}=p_{1}, \\
& -\frac{1}{A_{1}} \frac{d T_{13}}{d \xi_{1}}+k_{1} T_{11}=p_{3}, \\
& -\frac{1}{A_{1}} \frac{d M_{11}}{d \xi_{1}}+T_{13}=m_{1},
\end{aligned}
$$

where $\Omega_{2}=\left\{\xi: \xi=\left(\xi_{1}, \xi_{3}\right): \xi_{1}^{b} \leqslant \xi_{1} \leqslant \xi_{1}^{e},-\frac{h}{2} \leqslant \xi_{3} \leqslant \frac{h}{2}\right\}, T_{11}, T_{13}, M_{11}$ denote the forces and moment in the shell; $k_{1}\left(\xi_{1}\right)$ denotes the curvature of the shell; $A_{1}\left(\xi_{1}\right)$ denotes the Lame parameter of the shell. 
The forces $T_{11}, T_{13}$ and the moment $M_{11}$ in $\Omega_{2}$ using Hook's law can be represented as follows: $T_{11}=\frac{E_{2} h}{1-\nu_{2}^{2}} \varepsilon_{11}, T_{13}=k^{\prime} G^{\prime} h \varepsilon_{13}, M_{11}=\frac{E_{2} h^{3}}{12\left(1-\nu_{2}^{2}\right)} \chi_{11}$, where $G^{\prime}$ is the shear modulus, $k^{\prime}$ is the shear coefficient. On the other hand, strains $\varepsilon_{11}, \varepsilon_{13}, \chi_{11}$ can be represented as $\varepsilon_{11}=\frac{1}{A_{1}} \frac{d v_{1}}{d \xi_{1}}+k_{1} w, \varepsilon_{13}=$ $\frac{1}{A_{1}} \frac{d w}{d \xi_{1}}+\gamma_{1}-k_{1} v_{1}, \chi_{11}=\frac{1}{A_{1}} \frac{d \gamma_{1}}{d \xi_{1}}$.

Let $\sigma_{13}^{+}, \sigma_{33}^{+}$be the components of the surface forces vector that act on the outer $\left(\xi_{3}=\frac{h}{2}\right)$ and the inner $\left(\xi_{3}=-\frac{h}{2}\right)$ boundaries of the shell; let $m_{1}$ be the moment of external surface tension.

Then the external forces and moment in (1)-(3) that act on the shell are given by

$$
\begin{aligned}
p_{1} & =\left(1+k_{1} \frac{h}{2}\right) \sigma_{13}^{+}+\left(1-k_{1} \frac{h}{2}\right) \sigma_{13}^{-}, \\
p_{3} & =\left(1+k_{1} \frac{h}{2}\right) \sigma_{33}^{+}-\left(1-k_{1} \frac{h}{2}\right) \sigma_{33}^{-}, \\
m_{1} & =\frac{h}{2}\left(\left(1+k_{1} \frac{h}{2}\right) \sigma_{13}^{+}-\left(1-k_{1} \frac{h}{2}\right) \sigma_{13}^{-}\right) .
\end{aligned}
$$

Besides, for isotropic bodies $G^{\prime}=\frac{E_{2}}{2\left(1+\nu_{2}\right)}, k^{\prime}=\frac{5}{6}$.

Let us define $\Omega_{2}^{*}$ as the projection of $\Omega_{2}$ on the middle surface with $\xi_{3}=0$ and the interface $\Gamma_{I}$ between $\Omega_{1}$ and $\Omega_{2}$ as $\Gamma_{I}=\Gamma_{I_{1}} \cup \Gamma_{I_{2}} \cup \Gamma_{I_{3}}$, where $\Gamma_{I_{1}}=\left\{\xi: \xi=\left(\xi_{1}, \xi_{3}\right): \xi_{1} \in \Omega_{2 i n}^{*}, \xi_{3}=-\frac{h}{2}\right\}$; $\Gamma_{I_{2}}=\left\{\xi: \xi=\left(\xi_{1}, \xi_{3}\right): \xi_{1}=\xi_{1}^{b},-\frac{h}{2} \leqslant \xi_{3} \leqslant \frac{h}{2}\right\} ; \Gamma_{I_{3}}=\left\{\xi: \xi=\left(\xi_{1}, \xi_{3}\right): \xi_{1} \in \Omega_{2 i n}^{*}, \xi_{3}=\frac{h}{2}\right\}, \Omega_{2 i n}^{*}$ is the part of $\Omega_{2}^{*}$, that lies inside $\Omega_{1}$.

On the interfaces $\Gamma_{I}=\Gamma_{I_{1}} \cup \Gamma_{I_{2}} \cup \Gamma_{I_{3}}$ we assume the following contact conditions to hold: - on $\Gamma_{I_{1}}$ :

$$
\begin{gathered}
u_{n}=w, \quad u_{\tau}=-v_{1}+\frac{h}{2} \gamma_{1}, \\
\sigma_{n n}=-\sigma_{33}^{-}, \quad \sigma_{n \tau}=-\sigma_{13}^{-}
\end{gathered}
$$

- on $\Gamma_{I_{2}}$ :

$$
\begin{gathered}
u_{\tau}=w, \quad u_{n}=v_{1}+\xi_{3} \gamma_{1} \\
\int_{-\frac{h}{2}}^{\frac{h}{2}} \sigma_{n n} d \xi_{3}=T_{11}, \quad \int_{-\frac{h}{2}}^{\frac{h}{2}} \sigma_{n \tau} d \xi_{3}=T_{13} \\
\int_{-\frac{h}{2}}^{\frac{h}{2}} \xi_{3} \sigma_{n n} d \xi_{3}=M_{11}
\end{gathered}
$$

- on $\Gamma_{I_{3}}$ :

$$
\begin{gathered}
u_{n}=-w, \quad u_{\tau}=v_{1}+\frac{h}{2} \gamma_{1}, \\
\sigma_{n n}=-\sigma_{33}^{+}, \quad \sigma_{n \tau}=\sigma_{13}^{+} .
\end{gathered}
$$

Let $\Gamma_{1 D} \subset \Gamma_{1}, \Gamma_{1 N} \subset \Gamma_{1}, \Gamma_{1 N} \cup \Gamma_{1 D}=\varnothing, \Gamma_{1 D} \cup \Gamma_{I}=\varnothing, \Gamma_{1 N} \cup \Gamma_{I}=\varnothing$. Let $\Gamma_{2 D} \subset \Gamma_{2}, \Gamma_{2 N} \subset \Gamma_{2}$, $\Gamma_{2 N} \cup \Gamma_{2 D}=\varnothing, \Gamma_{2 D} \cup \Gamma_{I}=\varnothing, \Gamma_{2 N} \cup \Gamma_{I}=\varnothing$. As for the boundary conditions, we prescribe the following:

- on $\Gamma_{1 D}: u_{n}=u_{n}^{0}, u_{\tau}=u_{\tau}^{0}$;

- on $\Gamma_{1 N}: \sigma_{n n}=\sigma_{n n}^{0}, \sigma_{n \tau}=\sigma_{n \tau}^{0}$;

- on $\Gamma_{2 D}: v_{1}=v_{1}^{0}, w=w^{0}, \gamma_{1}=\gamma_{1}^{0}$;

- on $\Gamma_{2 N}: T_{11}=T_{11}^{0}, T_{13}=T_{13}^{0}, M_{11}=M_{11}^{0}$.

It is also possible to prescribe other kinds of boundary conditions, for example mixed boundary conditions. 


\section{Theoretical investigation of the model with thin inclusion}

In this section we describe the domain decomposition algorithm, which is used to numerically solve elasticity problems for the structures that contain thin covers or inclusions.

Let us denote by $\lambda=\left(\lambda_{11}, \lambda_{12}, \lambda_{21}, \lambda_{22}, \lambda_{23}, \lambda_{31}, \lambda_{32}\right)$ the unknown displacements on $\Gamma_{I}$. The domain decomposition algorithm is read as follows:

1) set an initial guess $\lambda^{0}$ for the unknown displacements on the interface $\Gamma_{I}$, set $\varepsilon>0$;

2 ) for $k=0,1, \ldots$ solve the boundary value problem in $\Omega_{1}$ with the displacements equal to $\lambda^{k}$ to obtain the approximation for the forces and momenta in $\Omega_{2}$ using (7)-(12);

3) solve the corresponding problem in $\Omega_{2}$ to find the displacements $u_{n}^{k}, u_{\tau}^{k}$ on $\Gamma_{I}$;

4) update the displacements $\lambda^{k}$ on $\Gamma_{I}$ :

- on $\Gamma_{I_{1}}$ :

$$
\begin{aligned}
& \lambda_{11}^{k+1}=(1-\theta) \lambda_{11}^{k}+\left.\theta u_{n}^{k}\right|_{\Gamma_{I_{1}}}, \\
& \lambda_{12}^{k+1}=(1-\theta) \lambda_{12}^{k}+\left.\theta u_{\tau}^{k}\right|_{\Gamma_{I_{1}}} ;
\end{aligned}
$$

- on $\Gamma_{I_{2}}$ :

$$
\begin{aligned}
& \lambda_{21}^{k+1}=(1-\theta) \lambda_{21}^{k}+\left.\theta v_{1}^{k}\right|_{\Gamma_{I_{2}}}, \\
& \lambda_{22}^{k+1}=(1-\theta) \lambda_{22}^{k}+\left.\theta w^{k}\right|_{\Gamma_{I_{2}}}, \\
& \lambda_{23}^{k+1}=(1-\theta) \lambda_{23}^{k}+\left.\theta \gamma_{1}^{k}\right|_{\Gamma_{I_{2}}} ;
\end{aligned}
$$

- on $\Gamma_{I_{3}}$ :

$$
\begin{aligned}
& \lambda_{31}^{k+1}=(1-\theta) \lambda_{31}^{k}+\left.\theta u_{n}^{k}\right|_{\Gamma_{I_{3}}}, \\
& \lambda_{32}^{k+1}=(1-\theta) \lambda_{32}^{k}+\left.\theta u_{\tau}^{k}\right|_{\Gamma_{I_{3}}},
\end{aligned}
$$

where $\theta>0$ is a relaxation parameter;

5) if $\left\|\lambda^{k+1}-\lambda^{k}\right\| \geqslant \varepsilon$, go to step 2, otherwise the algorithm ends.

Assume that on $\Gamma_{I}$ the function $\phi \in \Lambda$ is set, where $\Lambda$ is a linear space of the form

$$
\begin{aligned}
& \Lambda=\left\{\phi=\left(\phi_{1}, \phi_{2}, \phi_{3}\right): \phi_{1}=\left(\phi_{11}, \phi_{12}\right), \phi_{2}=\left(\phi_{21}, \phi_{22}, \phi_{23}\right), \phi_{3}=\left(\phi_{31}, \phi_{32}\right),\right. \\
&\left.\phi_{i j} \in H^{1}\left(\Gamma_{I_{i}}\right), i=1,3, j=1,2 ; \phi_{2 j} \in H^{1 / 2}\left(\Gamma_{I_{2}}\right), \phi_{2 j}=\text { const } \quad \text { on } \quad \Gamma_{I_{2}}, j=1,2,3\right\} .
\end{aligned}
$$

The scalar product and the norm in $\Lambda$ for $\phi, \psi \in \Lambda$ is defined as

$$
\begin{aligned}
(\phi, \psi)_{\Lambda}= & \int_{\Gamma_{I_{1}}}\left(\frac{d \phi_{11}}{d \xi_{1}} \frac{d \psi_{11}}{d \xi_{1}}+\frac{d \phi_{12}}{d \xi_{1}}\right) d \Gamma_{I_{1}}+\int_{\Gamma_{I_{1}}}\left(\phi_{11} \psi_{11}+\phi_{12} \psi_{12}\right) d \Gamma_{I_{1}} \\
& +\int_{\Gamma_{I_{2}}}\left(\phi_{21} \psi_{21}+\phi_{22} \psi_{22}+\phi_{23} \psi_{23}\right) d \Gamma_{I_{2}} \\
& +\int_{\Gamma_{I_{3}}}\left(\frac{d \phi_{31}}{d \xi_{1}} \frac{d \psi_{31}}{d \xi_{1}}+\frac{d \phi_{32}}{d \xi_{1}} \frac{d \psi_{32}}{d \xi_{1}}+\phi_{31} \psi_{31}+\phi_{32} \psi_{32}\right) d \Gamma_{I_{3}} ; \\
\|\phi\|_{\Lambda}= & (\phi, \phi)_{\Lambda}^{1 / 2} .
\end{aligned}
$$

The relationship between $\phi$ and the displacements on $\Gamma_{I}$ is given by

$$
\begin{array}{cc}
- \text { on } \Gamma_{I_{1}}: & \phi_{11}=u_{n}, \quad \phi_{12}=u_{\tau} ; \\
- \text { on } \Gamma_{I_{2}}: & \phi_{21}=v_{1}, \quad \phi_{22}=w, \quad \phi_{23}=\gamma_{1} ; \\
- \text { on } \Gamma_{I_{3}}: & \phi_{31}=u_{n}, \quad \phi_{32}=u_{\tau} .
\end{array}
$$


Let $S: \Lambda \rightarrow \Lambda^{*}$ be a Steklov-Poincare operator and $S_{i}$ be local Steklov-Poincare operators [6,10], that correspond to the domains $\Omega_{i}, i=1,2$, where $\Lambda^{*}$ is a space, conjugate to $\Lambda$.

The operators $S, S_{1}$ and $S_{2}$ are represented by

$$
\begin{aligned}
(S \phi, \psi)_{\Lambda}= & \left(S_{1} \phi, \psi\right)_{\Lambda}+\left(S_{2} \phi, \psi\right)_{\Lambda}, \\
\left(S_{1} \phi, \psi\right)_{\Lambda}= & \left\langle-A_{1}\left(1-k_{1} \frac{h}{2}\right) \sigma_{n n}(\phi), \psi_{11}\right\rangle_{\Gamma_{I_{1}}}+\left\langle-A_{1}\left(1-k_{1} \frac{h}{2}\right) \sigma_{n \tau}(\phi), \psi_{12}\right\rangle_{\Gamma_{I_{1}}} \\
& +\left\langle-A_{1}\left(1+k_{1} \frac{h}{2}\right) \sigma_{n n}(\phi), \psi_{31}\right\rangle_{\Gamma_{I_{3}}}+\left\langle-A_{1}\left(1+k_{1} \frac{h}{2}\right) \sigma_{n \tau}(\phi), \psi_{32}\right\rangle_{\Gamma_{I_{3}}} \\
& +\left\langle-\frac{1}{h} \int_{-h / 2}^{h / 2} \sigma_{n n}(\phi) d \xi_{3}, \psi_{21}\right\rangle_{\Gamma_{I_{2}}}+\left\langle-\frac{1}{h} \int_{-h / 2}^{h / 2} \sigma_{n \tau}(\phi) d \xi_{3}, \psi_{22}\right\rangle_{\Gamma_{I_{2}}} \\
& +\left\langle-\frac{1}{h} \int_{-h / 2}^{h / 2} \sigma_{n n}(\phi) \xi_{3} d \xi_{3}, \psi_{23}\right\rangle_{\Gamma_{I_{2}}} ; \\
\left(S_{2} \phi, \psi\right)_{\Lambda}= & \left\langle-A_{1}\left(1-k_{1} \frac{h}{2}\right) \sigma_{33}^{-}(\phi), \psi_{11}\right\rangle_{\Gamma_{I_{1}}}+\left\langle-A_{1}\left(1+k_{1} \frac{h}{2}\right) \sigma_{33}^{+}(\phi), \psi_{31}\right\rangle_{\Gamma_{I_{3}}} \\
& +\left\langle-A_{1}\left(1-k_{1} \frac{h}{2}\right) \sigma_{13}^{-}(\phi), \psi_{12}\right\rangle_{\Gamma_{I_{1}}}+\left\langle A_{1}\left(1+k_{1} \frac{h}{2}\right) \sigma_{13}^{+}(\phi), \psi_{32}\right\rangle_{\Gamma_{I_{3}}} \\
& +\left\langle\frac{1}{h} T_{11}(\phi), \psi_{21}\right\rangle_{\Gamma_{I_{2}}}+\left\langle\frac{1}{h} T_{13}(\phi), \psi_{22}\right\rangle_{\Gamma_{I_{2}}}+\left\langle\frac{1}{h} M_{11}(\phi), \psi_{23}\right\rangle_{\Gamma_{I_{2}}}
\end{aligned}
$$

where $\langle u, v\rangle_{\Gamma_{I}}$ is a bilinear form that can be formally written as

$$
\langle u, v\rangle_{\Gamma_{I}}=\int \Gamma_{I} u v d \Gamma_{I}, \quad u \in H^{1 / 2}\left(\Gamma_{I}\right), \quad v \in\left(H^{1 / 2}\left(\Gamma_{I}\right)\right)^{*} .
$$

We now investigate the properties of the local Steklov-Poincare operators $S_{1}$ and $S_{2}$.

Lemma 1. Operator $S_{1}$ is linear, continuous, symmetric and elliptic on $\Lambda$.

Proof. Follows from the corresponding properties of the Steklov-Poincare operators for the classical problem of linear elasticity and continuous embedding [9,10]: $H^{1}\left(\Gamma_{I_{i}}\right) \subset \subset H^{1 / 2}\left(\Gamma_{I_{i}}\right), i=1,2,3$.

Lemma 2. Operator $S_{2}$ is linear, symmetric, continuous and elliptic on $\Lambda$.

Proof. Expressing functions $\phi_{i j}$ in terms of displacements using (13)-(15), we write

$$
\begin{aligned}
\left(S_{2} \phi, \psi\right)_{\Lambda}= & \left(A_{1}\left(\left(1+k_{1} \frac{h}{2}\right) \sigma_{33}^{+}(\phi)-\left(1-k_{1} \frac{h}{2}\right) \sigma_{33}^{-}\right), \tilde{w}\right)_{\Omega_{2}^{*}} \\
& +\left(A_{1}\left(\left(1+k_{1} \frac{h}{2}\right) \sigma_{13}^{+}(\phi)+\left(1-k_{1} \frac{h}{2}\right) \sigma_{13}^{-}\right), \tilde{v}_{1}\right)_{\Omega_{2}^{*}} \\
& +\left(A_{1} \frac{h}{2}\left(\left(1+k_{1} \frac{h}{2}\right) \sigma_{13}^{+}(\phi)-\left(1-k_{1} \frac{h}{2}\right) \sigma_{13}^{-}\right), \tilde{\gamma}_{1}\right)_{\Omega_{2}^{*}} \\
& +\left\langle\frac{1}{h} T_{11}, \tilde{v}_{1}\right\rangle_{\Gamma_{I_{2}}}+\left\langle\frac{1}{h} T_{13}, \tilde{w}\right\rangle_{\Gamma_{I_{2}}}+\left\langle\frac{1}{h} M_{11}, \tilde{\gamma}_{1}\right\rangle_{\Gamma_{I_{2}}},
\end{aligned}
$$

where $(u, v)_{\Omega_{2}^{*}}=\int_{\xi_{1 b}}^{\xi_{1 e}} u v d \xi_{1}$ for $u, v \in L_{2}\left(\Omega_{2}^{*}\right)$.

Substituting the expressions for $\sigma_{i j}^{*}$ from (1)-(6) into (16), integrating by parts, we reduce the properties of the operator $S_{2}$ to the properties of the operator of the weak formulation of the problem on the middle line. 
It is known that the operator of the shell theory (1)-(3) is linear, elliptic, symmetric and continuous $[5,6,16]$.

Therefore, we obtain

$$
\left(S_{2} \phi, \phi\right)_{\Lambda} \geqslant c^{2} \int_{\xi_{1 b}}^{\xi_{1 e}}\left(\left(\frac{d v_{1}}{d \xi_{1}}\right)^{2}+\left(\frac{d w}{d \xi_{1}}\right)^{2}+\left(\frac{d \gamma_{1}}{d \xi_{1}}\right)^{2}+v_{1}^{2}+w^{2}+\gamma_{1}^{2}\right) d \xi_{1}, c \neq 0
$$

From the expression above using trace inequality [10] we get

$$
\begin{aligned}
\left(S_{2} \phi, \phi\right)_{\Lambda} \geqslant & c_{1}^{2} \int_{\xi_{1 b}}^{\xi_{1 e}}\left(\left(\frac{d w}{d \xi_{1}}\right)^{2}+\left(-\frac{d v_{1}}{d \xi_{1}}+\frac{h}{2} \frac{d \gamma_{1}}{d \xi_{1}}\right)^{2}+w^{2}+\left(-v_{1}+\frac{h}{2} \gamma_{1}\right)^{2}\right) d \xi_{1}+c_{2}^{2}\left\|\phi_{2}\right\|_{H^{1 / 2}\left(\Gamma_{I_{2}}\right)} \\
& +\int_{\xi_{1 b}}^{\xi_{1 e}}\left(\left(\frac{d w}{d \xi_{1}}\right)^{2}+\left(\frac{d v_{1}}{d \xi_{1}}+\frac{h}{2} \frac{d \gamma_{1}}{d \xi_{1}}\right)^{2}+w^{2}+\left(v_{1}+\frac{h}{2} \gamma_{1}\right)^{2}\right) d \xi_{1}, \quad c_{i} \neq 0, \quad i=1,2,3 .
\end{aligned}
$$

Since the operator of the shell theory problem (1)-(3) is continuous, the estimate

$$
\begin{aligned}
\left|\left(S_{2} \phi, \psi\right)_{\Lambda}\right| & \leqslant C\left(\int_{\xi_{1 b}}^{\xi_{1 e}}\left(\left(\frac{d v_{1}}{d \xi_{1}}\right)^{2}+\left(\frac{d w}{d \xi_{1}}\right)^{2}+\left(\frac{d \gamma_{1}}{d \xi_{1}}\right)^{2}+v_{1}^{2}+w^{2}+\gamma_{1}^{2}\right)^{1 / 2}\right. \\
& \left(\int_{\xi_{1 b}}^{\xi_{1 e}}\left(\left(\frac{d \tilde{v}_{1}}{d \xi_{1}}\right)^{2}+\left(\frac{d \tilde{w}}{d \xi_{1}}\right)^{2}+\left(\frac{d \tilde{\gamma}_{1}}{d \xi_{1}}\right)^{2}+\tilde{v}_{1}^{2}+\tilde{w}^{2}+\tilde{\gamma}_{1}^{2}\right)^{1 / 2}, \quad C>0\right.
\end{aligned}
$$

is valid.

Using arguments, similar to the ones in the first part of the proof, we conclude that the operator $S_{2}$ is continuous on $\Lambda$.

It can be shown, that the Steklov-Poincare equation has a unique solution on $\Lambda$. The proof directly follows from Lax-Milgram lemma.

Theorem 3. The sequential Dirichlet-Neumann domain decomposition algorithm presented in this section is convergent to the solution of the corresponding Steklov-Poincare equation.

Proof. The proof follows from the properties of local Steklov-Poincare operators and theorem about convergence of Dirichlet-Neumann domain decomposition schemes [17].

\section{Numerical experiments}

\subsection{Girkmann type problems [14]}

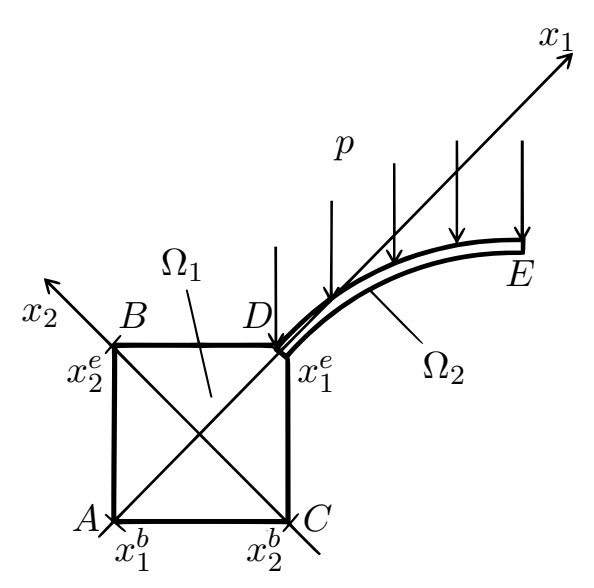

Fig. 2. Physical structure under consideration.
Let $\Omega_{1}$ be a polygon with $x_{1}^{b}=-1, x_{2}^{b}=-1, x_{1}^{e}=1, x_{2}^{e}=1$. To the main part in $\Omega_{1}$ a thin body in $\Omega_{2}$ is attached on its edge. The thickness of the body in $\Omega_{2}$ is $h=0.01$ (Fig. 2).

On the boundaries $A C$ and $A B$ the structure is fixed (the displacements are equal to zero); we prescribe a load of $p=$ $1 \mathrm{~Pa} / \mathrm{m}$ on the outer boundary of the body in $\Omega_{2}$ (Fig. 2 ); on the edge with the point $E$ the symmetry conditions are set; all the other parts of the outer boundary are traction-free.

We consider the following physical parameters of the bodies: Young's modulus of the main part in $\Omega_{1}$ is equal to $E_{1}=25000 \mathrm{MPa}$, which corresponds to concrete; the Young's modulus of the thin part in $\Omega_{2}$ is equal to $E_{2}=20580 \mathrm{MPa}$, which corresponds to cork. Poisson's ratio of the body in $\Omega_{1}$ is equal to $\nu_{1}=0.33$, in $\Omega_{2}-\nu_{2}=0$. 
For the numerical solution we use FEM in the shell with bubble shape functions. For the main part we use boundary element method with quadratic shape functions. Problems in both parts are connected using domain decomposition algorithm (DirichletNeumann scheme) [17].

In all the cases under consideration the convergence is obtained in around 5 iterations. The results correspond to a case of 202 boundary elements, 32 finite elements of the fourth order. It should be noted that the mesh refinement or the change of the order of the shape functions do not change the solution significantly.

Let us consider different cases of the curve shapes that describe middle line of the body in $\Omega_{2}$ : circle arc, parabola and chain curve. The unknown coefficients of the parametric representation of the curves are chosen in such a way that all the curves have the same endpoints $D$ and $E$. Moreover, all the curves are symmetric with respect to the axis, which passes through the point $E$ and is colinear to $A B$.

The parametric representations for the circle arc (a), parabola (b) and chain curve (c) take the following forms:

(a):

$$
\begin{aligned}
& x_{1}(\alpha)=R \sin \alpha, \\
& x_{2}(\alpha)=R \cos \alpha, \quad \frac{\pi}{4} \leqslant \alpha \leqslant \frac{\pi}{2} ;
\end{aligned}
$$

$$
\begin{aligned}
& x_{1}(\alpha)=-\frac{2-\sqrt{2}}{R} x_{2}^{2}+R, \\
& x_{2}(\alpha)=R \cos \alpha, \quad \frac{\pi}{4} \leqslant \alpha \leqslant \frac{\pi}{2}
\end{aligned}
$$

$$
\begin{aligned}
& x_{1}(\alpha)=-\frac{4.497}{2}\left(e^{\frac{x_{2}}{4.497}}+e^{-\frac{x_{2}}{4.497}}\right)+9.502, \\
& x_{2}(\alpha)=R \cos \alpha, \quad \frac{\pi}{4} \leqslant \alpha \leqslant \frac{\pi}{2},
\end{aligned}
$$

with $R=5.005$.

The graphs of three curves are shown in Fig. 3.

We can conclude from Fig. 3 that the graphs of the curves lie close to each other.

Formulae for the calculation of Lame parameter $A_{1}$ and curvature $k_{1}$ of the middle line of the shells have the form

$$
\begin{aligned}
& A_{1}=\sqrt{x_{1}^{\prime 2}+x_{2}^{\prime 2}}, \\
& k_{1}=\frac{x_{1}^{\prime \prime} x_{2}^{\prime}-x_{1}^{\prime} x_{2}^{\prime \prime}}{A_{1}^{3}} .
\end{aligned}
$$

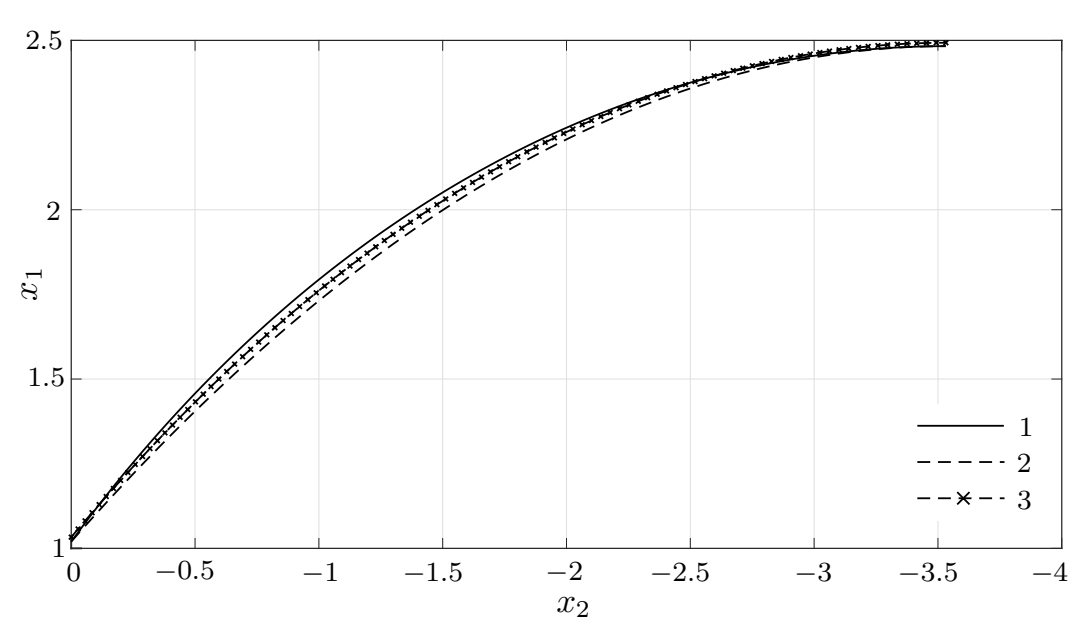

Fig. 3. Middle Line of Different Curves.

Let us calculate the stress-strain state for the body depicted on the Fig. 2 .

Fig. 4 shows the displacements in the case of the chain curve as a middle line of $\Omega_{2}$ for different meshsizes, Fig. 5 shows the momenta that arise on the middle line of $\Omega_{2}$.

Curve 1 on the Figs. 4-5 corresponds to the case of 8 fourth order elements with bubble basis functions in $\Omega_{2}$ and 42 quadratic boundary elements in $\Omega_{1}$, curve $2-16$ elements in $\Omega_{2}$ and 74 elements in $\Omega_{1}$, curve $3-32$ elements in $\Omega_{2}$ and 138 elements in $\Omega_{1}$, curve $4-64$ elements in $\Omega_{2}$ and 202 elements in $\Omega_{1}$. 


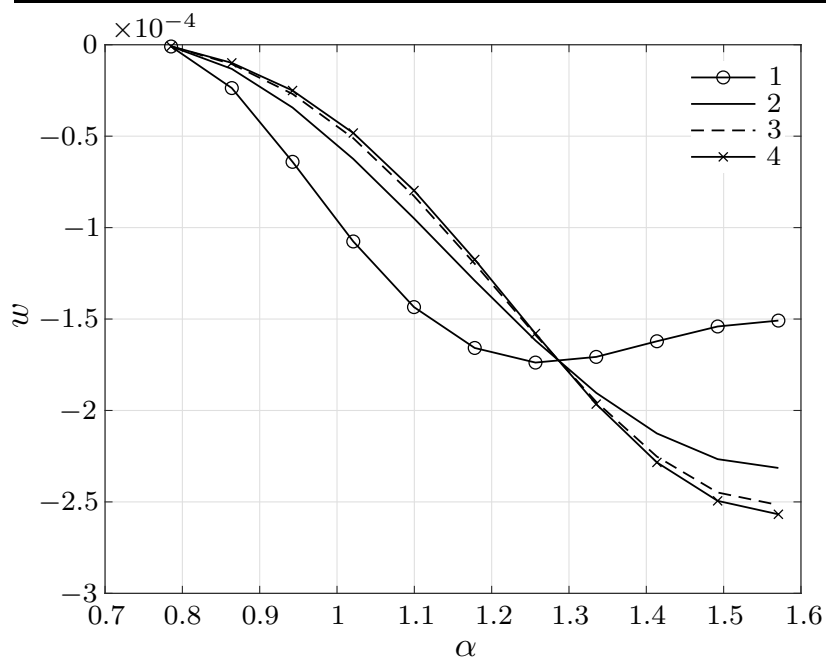

Fig. 4. Displacements $w$ on the middle line of the shell in the case of different meshsizes.

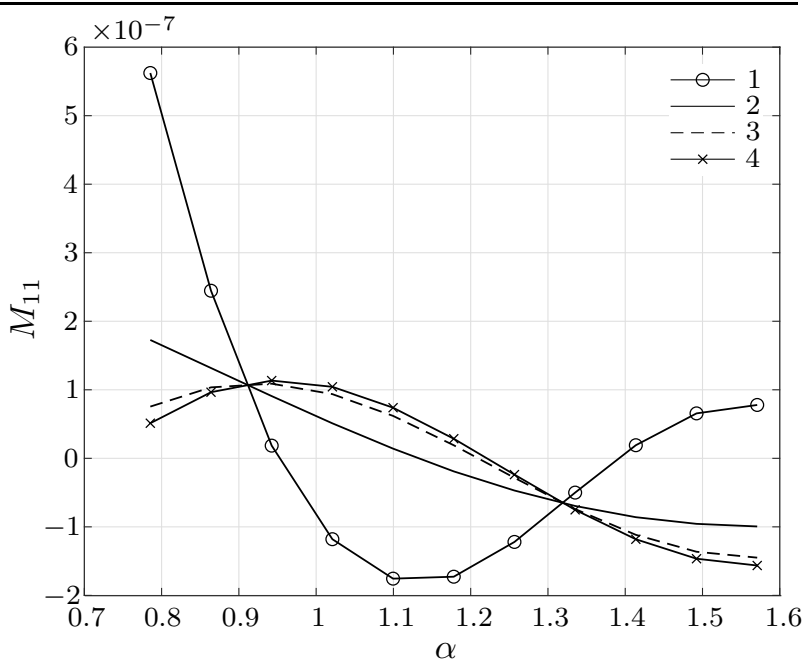

Fig. 5. Moment $M_{11}$ on the middle line of the shell in the case of different meshsizes.

On the interface $0 \leqslant x_{2} \leqslant h, x_{1}=x_{1}^{e}$ we have to set the Neumann condition for the problem in main part and Dirichlet condition for the problem in the shell. The displacements on the interface for the shell are found using the conditions

$$
\begin{aligned}
& u_{n}=v_{1}+\xi_{3} \gamma_{1}, \\
& u_{\tau}=w .
\end{aligned}
$$

Applying the first condition at the points $\xi_{3}=0$ and $\xi_{3}=h / 2$, we obtain:

$$
\begin{aligned}
\left.v_{1}\right|_{\xi_{1}=0} & =-\left.u_{n}\right|_{\xi_{3}=0}, \\
\left.\gamma_{1}\right|_{\xi_{1}=0} & =\frac{2}{h}\left(\left.u_{n}\right|_{\xi_{3}=\frac{h}{2}}-\left.u_{n}\right|_{\xi_{3}=0}\right) .
\end{aligned}
$$

Similarly, when the second condition is applied at the point $\xi_{3}=0$, the results are as follows:

$$
\left.w\right|_{\xi_{1}=0}=\left.u_{\tau}\right|_{\xi_{3}=0} .
$$

Let us consider the conditions on the loads that need to be imposed on the interface for the problem in the main part. In order to express $\sigma_{n \tau}$ and $\sigma_{n n}$ we use the conditions stated below.

For $\sigma_{n \tau}$ :

$$
\begin{aligned}
\int_{-\frac{h}{2}}^{\frac{h}{2}} \sigma_{n \tau} d \xi_{3} & =T_{13}, \quad \sigma_{n \tau}\left(\xi_{3}\right)=\left.\sigma_{13}^{-}\right|_{\xi_{1}=0}, \\
\sigma_{n \tau}\left(\xi_{3}\right) & =-\left.\sigma_{13}^{+}\right|_{\xi_{1}=0}
\end{aligned}
$$

for $\sigma_{n n}$ :

$$
\int_{-\frac{h}{2}}^{\frac{h}{2}} \sigma_{n n} d \xi_{3}=T_{11}, \quad \int_{-\frac{h}{2}}^{\frac{h}{2}} \sigma_{n n} \xi_{3} d \xi_{3}=M_{11} .
$$

Let us assume that on the interface $\sigma_{n \tau}=a \xi_{3}^{2}+b \xi_{3}+c, \sigma_{n n}=e \xi_{3}+f$, where $a, b, c, e, f$ are the unknown coefficients. These assumptions are based on the fact, that we have three conditions for $\sigma_{n \tau}$ and two conditions on $\sigma_{n n}$.

The computations yield

$$
\sigma_{n n}\left(\xi_{3}\right)=M_{11} \frac{12}{h^{3}} \xi_{3}+\frac{T_{11}}{h},
$$




$$
\begin{aligned}
\sigma_{n \tau}= & \left(\frac{3}{h^{2}}\left(\left.\sigma_{13}^{-}\right|_{\xi_{1}=0}-\left.\sigma_{13}^{+}\right|_{\xi_{1}=0}\right)-\frac{6}{h^{3}} T_{13}\right) \xi_{3}^{2}-\frac{1}{h}\left(\left.\sigma_{13}^{-}\right|_{\xi_{1}=0}+\left.\sigma_{13}^{+}\right|_{\xi_{1}=0}\right) \xi_{3} \\
& +\frac{1}{h}\left(T_{13}-\frac{1}{4}\left(h\left(\left.\sigma_{13}^{-}\right|_{\xi_{1}=0}-\left.\sigma_{13}^{+}\right|_{\xi_{1}=0}\right)-2 T_{13}\right) .\right.
\end{aligned}
$$

It is shown (see for example [16]) that the smallest displacement in the normal direction is achieved when the middle line of the thin part of the body is a chain curve. The largest displacement in the normal direction arises when the middle line of the thin part is a circle segment.

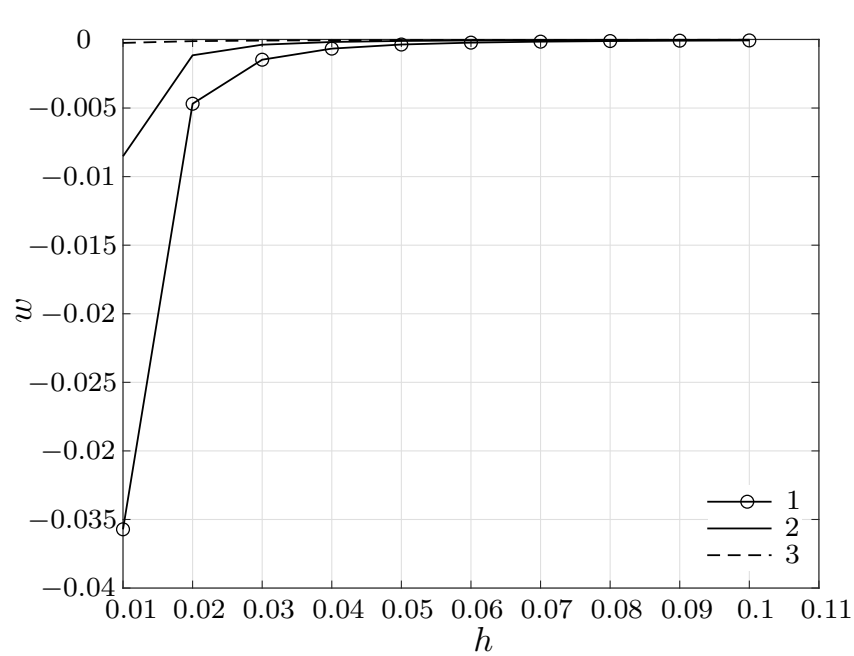

Fig. 6. Displacements $w$ on the middle line of the shell depending on its thickness.

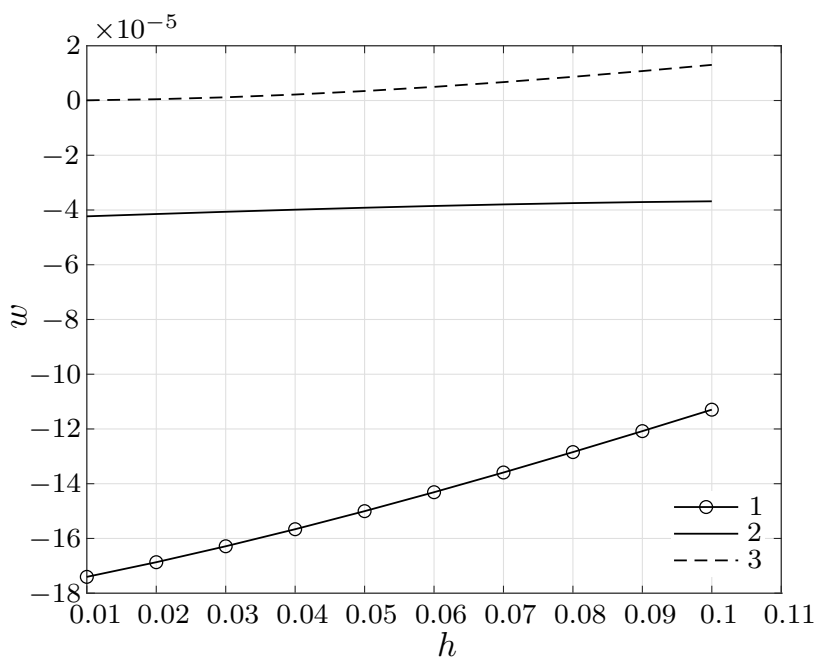

Fig. 7. Moment $M_{11}$ on the middle line of the shell depending on its thickness.

Figs. 6-7 show the dependence of displacements and momenta on the thickness of the shell, curve 1 corresponds to middle line of the shell being a circular segment, curve 2 - part of the parabola, curve 3 - chain curve.

In conclusion, the stress-strain state of the thin part of the structure in the case of the Girkmann problem heavily depends on the geometrical parameters of the middle line of the shell (shape, curvature) as well as its thickness.

\subsection{A body with an inclusion}

Consider a numerical example that illustrates the application of the proposed algorithm to the approximate determination of the stress-strain state of a body with an inclusion. Let the domain $\Omega=\Omega_{1} \cup \Omega_{2}$ be a rectangle with vertices at the points $\left(x_{1}^{b}, x_{2}^{b}\right),\left(x_{1}^{b}, x_{2}^{e}\right),\left(x_{1}^{e}, x_{2}^{e}\right)$, and $\left(x_{1}^{e}, x_{2}^{b}\right)$, and let the domain $\Omega_{2}$ be a rectangle with vertices at the points $\left(x_{1}^{m}, x_{2}^{m_{1}}\right),\left(x_{1}^{m}, x_{2}^{m_{2}}\right),\left(x_{1}^{e}, x_{2}^{m_{2}}\right)$, $\left(x_{1}^{e}, x_{2}^{m_{1}}\right)$, where $x_{1}^{b}=0.5, x_{1}^{m}=1, x_{2}^{m_{1}}=x_{2}^{m}-\frac{h}{2}$, $x_{2}^{m_{2}}=x_{2}^{m}+\frac{h}{2}, x_{1}^{e}=2, x_{2}^{b}=0$, and $x_{2}^{e}=1$ (Fig. 8). Here, all sizes of the body are referred to the dimensions.

In order to compare the results, we consider

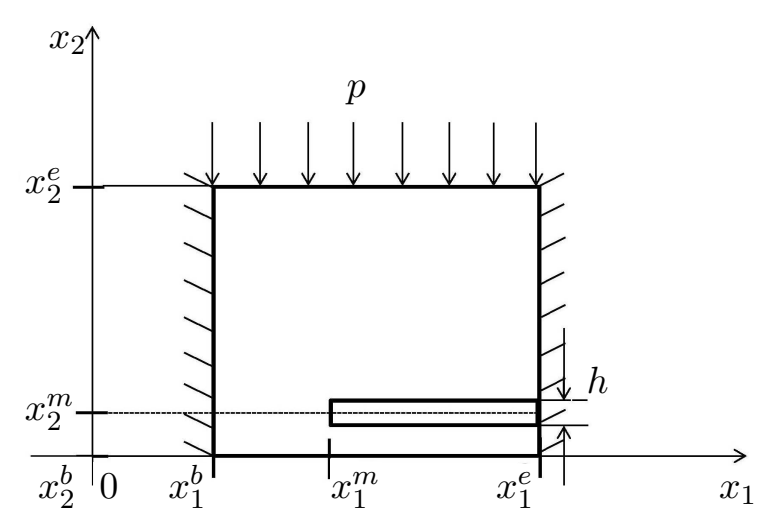

Fig. 8. Body with inclusion. several cases of structures with inclusions of different thicknesses:

1) $h=0.1\left(x_{2}^{m}=0.15\right)$.

2) $h=0.05\left(x_{2}^{m}=0.125\right)$.

3) $h=0.02\left(x_{2}^{m}=0.11\right)$. 
Along the straight lines $x_{1}=x_{1}^{b}$ and $x_{1}=x_{1}^{e}$ the structure is fixed, and along the straight line $x_{2}=x_{2}^{e}$ it is loaded by the uniform normal force $p=1 \mathrm{MPa}$; the boundary of the domain $\Omega$ lying on $x_{2}=x_{2}^{b}$ is free. Young's modulus of the material of the body $\Omega_{1}$ is equal to $E_{1}=3300 \mathrm{MPa}$, which corresponds to acryl, and the material of the inclusion $\Omega_{2}$ is glass fiber with $E_{2}=80000 \mathrm{MPa}$. Poisson's ratios of the materials of the body $\Omega_{1}$ and inclusion $\Omega_{2}$ are equal to $\nu_{1}=0.37$ and $\nu_{2}=0.22$ respectively.

In the figure Fig. 9 the comparison for the displacements $u_{2}$ along the line $x_{2}=x_{2}^{b}$ in the case 2 is shown between the numerical solution obtained using the approach from section 3 (curve 1) and the numerical solution obtained using COMSOL 5.0 (curve 2). In our approach we use 116 second order elements inside the main part (476 degrees of freedom) and 16 elements with fourth order bubble functions (194 degrees of freedom). The solutions in COMSOL 5.0 uses 1311 triangles with 5428 degrees of freedom. From Fig. 9 we can conclude that the domain decomposition approach produces reliable numerical solutions.

Taking into account the linearity of the problem, we solved approximately the problem in the domain $\Omega_{1}$ only in the first iteration, and in subsequent iterations, the approximate solution of the BEM was constructed as a linear combination of already known solutions.

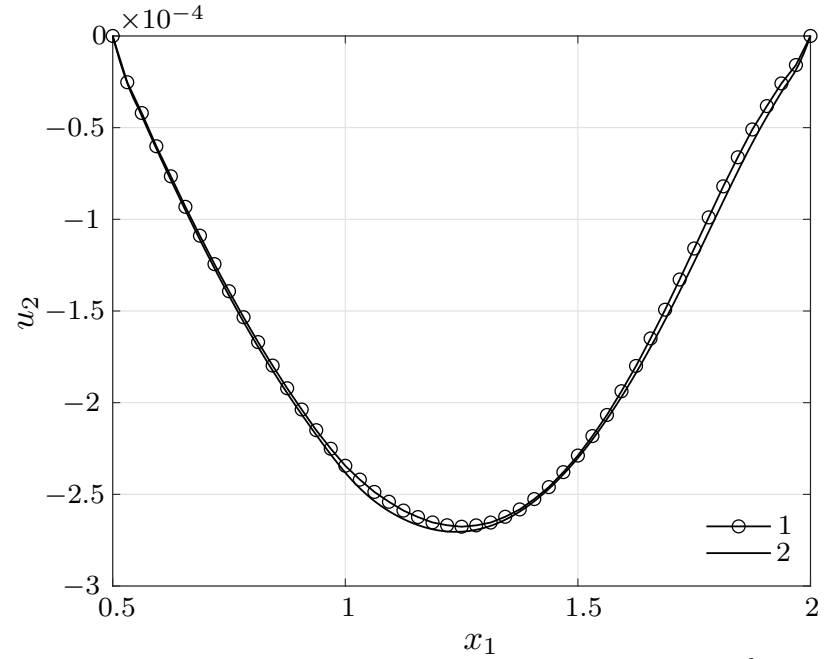

Fig. 9. Displacement $u_{2}$ on the curve $x_{2}=x_{2}^{b}$.

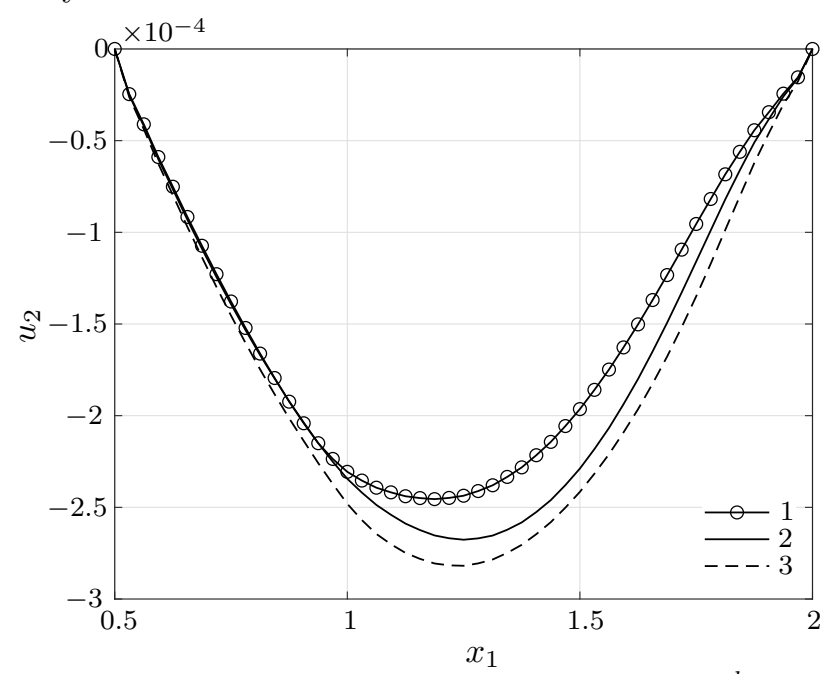

Fig. 10. Displacement $u_{2}$ on the curve $x_{2}=x_{2}^{b}$ depending on the thickness of the shell.

In Fig. 10, the comparison for the displacements $u_{2}$ along the line $x_{2}=x_{2}^{b}$ for the 3 cases $(1,2$ and 3) is shown (curves 1, 2 and 3 respectively). For $h=0.1$ and $h=0.05$ we use 116 second order elements inside the main part and 16 elements with fourth order bubble functions, for $h=0.02114$ second order elements inside the main part and 16 elements with fourth order bubble functions are used. Since the physical material of the inclusion is harder than the main part of the structure, the displacements become larger as the thickness of the inclusion decreases.

\section{Concluding remarks}

The approach under analysis allows to reduce computational cost due to the use of multiscale approach. The space dimension of each subproblem is reduced by one: in a matrix part it is achieved with the use of BEM and in a thin inclusion it is achieved with the help of Timoshenko shell theory.

The numerical schemes based on the domain decomposition method have been theoretically investigated with the Steklov-Poincare operators and the theoretical results have been obtained.

Our approach, obviously, has its limitations (BEM can be applied only for the homogeneous matrix, shell theory requires the corresponding assumptions for stresses and displacements to hold). However, it is efficient for thin inclusions of a certain thickness (e.g., in case of inclusion 1/10 - 1/50 of the thickness of matrix). Besides, it provides quite a simple way for evaluating the impact of thin inclusion 
or coating on the stress-strain state of the matrix. From this prospective, the model may be applied for solving the engineering problems.

It must be pointed out that the proposed approach may be applied to similar problems in other modeling applications as well as for more complex problems (e.g. the ones that require nonlinear shell theory for the description of thin inclusions or several inclusions).

[1] Quarteroni A. Multifields modeling in numerical simulation on partial differential equations. GAMMMitteilungen. 19 (1), 45-63 (1996).

[2] Savula Ya. H., Dyyak I. I., Krevs V. V. Heterogeneous mathematical models in numerical analysis of structures. Int. journ. computers \& mathematics with applications. 42 (8-9), 1201-1216 (2001).

[3] Wang F. Y., Xu Y. L., Qu W. L. Mixed-dimensional finite element coupling for structural multi-scale simulation. Finite elements in analysis and design. 92, 12-25 (2014).

[4] Wellmann Ch., Wriggers P. A two-scale model of granular materials. Comput. methods appl. mech. engrg. 205-208, 46-58 (2012).

[5] Savula Ya., Mang H., Dyyak I., Pauk N. Coupled boundary and finite element analysis of a special class of 2D problems of the theory of elasticity. Comput. \& struct. 75 (2), 157-165 (2000).

[6] Dyyak I., Savula Ya., Shahin M. Investigation of the heterogeneous problems of the elasticity with coupled boundary-finite element schemes. Advances in applied and computational mathematics. New York, Nova science publishers inc. p. 47 (2006).

[7] Vynnytska L., Savula Ya. Mathematical modeling and numerical analysis of elastic body with thin inclusion. Computational mechanics. 50 (5), 533-542 (2012).

[8] Savula Ya. H., Dyyak I. I., Dubovik A. V. Use of a combination model to calculate the stress-strain state of three-dimensional structures. Sov. appl. mech. 25 (9), 904-909 (1989).

[9] Ciarlet P. G. Plates and junctions in elastic multi-structures. Paris, Springer-Verlag (1990).

[10] Hsiao G. C., Wendland W. L. Boundary integral equations. Berlin, Springer-Verlag (2008).

[11] Haas M., Kuhn G. Mixed-dimensional, symmetric coupling of FEM and BEM. Engineering analysis with boundary elements. 27 (6), 575-582 (2003).

[12] Savula Ya., Dyyak I. D-adaptive model for the elasticity problem. Computer assisted mechanics \& engineering sciences. 5 (1), 65-74 (1998).

[13] Toselli A., Widlund O. Domain decomposition methods - algorithms and theory. Berlin, Springer-Verlag (2005).

[14] Pitkaranta J., Babuska I., Szabo B. The dome and the ring: verification of an old mathematical model for the design of a stiffened shell roof. Computers and mathematics with applications. 64 (1), 48-72 (2012).

[15] Pelekh B. L. Generalized shell theory. Lviv, Vyshcha Shkola (1978), (in Russian).

[16] Styahar A. Numerical investigation of the Girkmann problem with FEM/BEM coupling using domain decomposition. Journal of numerical and applied mathematics. 116 (2), 141-151 (2014).

[17] Quarteroni A., Valli A. Domain decomposition methods for partial differential equations. Oxford, Oxford Univ. Press (1999). 


\title{
Числовий аналіз різномасштабної математичної моделі пружного тіла з тонким включенням комбінованими МГЕ та МСЕ
}

\author{
Дияк I. I. ${ }^{1}$, Рубіно Б. ${ }^{2}$, Савула Я. Г. ${ }^{1}$, Стягар А. О. ${ }^{1,2}$ \\ 1 Львівський національний університет імені Івана Франка, \\ вул. Університетсъка, 1, Львів, 79000, Україна \\ ${ }^{2}$ Університет міста Л'Аквіли, \\ вул. Ветойо, Коппіто, Л'Аквіла, 67100, Італія
}

Статтю присвячено математичному моделюванню різномасштабних пружних конструкцій, що складаються із матриці та тонких покриттів або включень. Матрицю описано рівняннями класичної теорії пружності, для опису тонких частин конструкції використано теорію оболонок Тимошенка. На спільній границі між середовищами задано ідеальні умови контакту. Розроблено алгоритм на основі методу граничних елементів у матриці та методу скінченних елементів високого порядку в тонких частинах конструкції. Ці два методи поєднано за допомогою методу декомпозиції областей. Для ілюстрації запропонованого підходу розглянуто два числові приклади: задачу типу Гіркмана і задачу для пружного тіла з тонким включенням. У першому прикладі проаналізовано залежність переміщень і напружено-деформованого стану від форми оболонки, у другому - залежність переміщень від товщини включення.

Ключові слова: метод скінченних елементів, метод граничних елементів, теорія оболонок Тимошенка, теорія пружності.

2000 MSC: 74-xx; 65N30; 65N38; 65N55

УдК: $531 ; 519.6$ 